\title{
The crack quasi-static growth and analysis of interlaminar crack resistance of layered composite
}

\author{
Igor Pavelko' ${ }^{1}$, Vitalijs Pavelko² \\ ${ }^{1}$ Riga Technical College, Physics Department, LV1084 Riga, Latvia \\ ${ }^{2}$ Riga Technical University, Institute of Aeronautics, LV1003 Riga, Latvia
}

\begin{abstract}
Using the model of quasi-static crack growth and test result of the double cantilever beam (DCB) sample of layred carbon/epoxy composite, the general regularities of the interlaminar crack resistance of mode 1 were studied. The main attention was focused on the variability of crack resistance associated, on the one hand, with the continuity of the fracture process, and, on the other hand, with the non-homogeneity of the material structure, which causes local random deviations from the average characteristics of the material.The dissipation energy rate (total crack resistance) and crack resistance function ( $R$-curve) were extracted from test results and their properties analyzed.
\end{abstract}

\section{Introduction}

The quasi-static growth of a crack is the continuous process of an infinitely slow transition from one equilibrium state to another during which the parameters of any intermediate state satisfy to equation of energy balance. In a case of a crack growth the main internal parameter of an elastic body, strain energy, changes as results of external loading and an increment of crack surface.

In contrast to the classic theory of brittle fracture [1] and its quasi-brittle versions [2,3] the concept of quasi-static growth of a crack allows to describe of crack propagation as the continuos process begining from the start of external loading.

There were numerous attempts of analytical description of the stable growth of the crack under increasing load. Critical analysis of the most popular of them was done [4]. Apparently, the first attempt of a semi-empirical description was done in [5]. The most perspective are the models based on the energy balance equation. Using the Dugdale's model of plastic zone and corresponding estimate of the energy dissipation rate, in [6] there was obtained a nonlinear differential equation for 'load/crack length' function. Solution of this equation describes of a crack continuos growth in the Griffith-type configuration of infinite quasibrittle plate. In the approximate analysis [7] the dissipation energy rate is presented as some sum of two parts. One of them is defined by the external load increase at the fixed size of a crack and other one by the crack size growth at the constant load. This introduction of the energy dissipation rate allows to describe the stable growth of a crack. The energy balance aproarch were used also by [8-14]. Comparison of some mentioned energy-based models is done in [15]. The thermodynamics of crack growth in elastic-plastic materials is developed in $[16,17]$ and it is shown an approach of computer-aided simulation of crack growth under condition of total energy balance.

A simplified one-parameter description of quasi-static crack growth is given by [18] for the case of the small-scale cohesive zone. The improved version of a model is presented in [19]. The main advantage of this model is invariance of the governing differential equation in respect of geometrical configuration and the means of loading. 
In general, the quasi-static growth of a crack is interpreted as the continuous process of an infinitesimal transition from one equilibrium state to another during which the parameters of any state satisfied to equation of energy balance. In the last paper[19] there is considered some application of model for damage reconstruction in the double-cantilever beam (DCB) sample of a layered composite. Important consequence of this research is the conclusion on non-homogeneity of the crack resistance and the interlaminar fracture toughness of this material.

In the presented paper the investigation is focused to non-homogeneity of the crack resistance using the developed model of quasy-static growth of a crack.

\section{On some regularities of the interlaminar destruction of layered composite}

The DCB-sample is the most popular type for measurement of interlaminar fracture toughness of composite. The standard testing of the unidirectional carbon/epoxy layered composite was done for measurement of the interlaminar crack resistance properties of this material. The quasi-static tests of DCB sample (Figure 1,a), according to the standard [20], were carried out on an Instron 8800 hydraulic testing machine with controlled extension at a constant rate of $5 \mathrm{~mm} / \mathrm{min}$. To increase the accuracy of measurement of small values of the load, an S2M meter of small loads (HBM Test and Measurement), with the upper measurement limit of $1 \mathrm{kN}$, was connected in series in the loading circuit as the basic force transducer (Figure 1,b). After each jump-like growth in the size of delamination, the loading was interrupted, the crack (delamination) length was visually registered (Fig. 3b) using lowsensitive microscope (x10), and loading was continued at the next stage. This procedure allowed us to obtain the full relation between the opening of delamination and the corresponding load at each registered crack length.

Theoretically for the perfect elastic-plastic material with small scale cohesive zone the quasi-static process of a crack (interlaminar delamination) growth should be monotonic and stable for both ascending and descending branches. This procedure allowed to obtain the full relation between the opening of delamination (extension) and the corresponding load at each registered crack length.

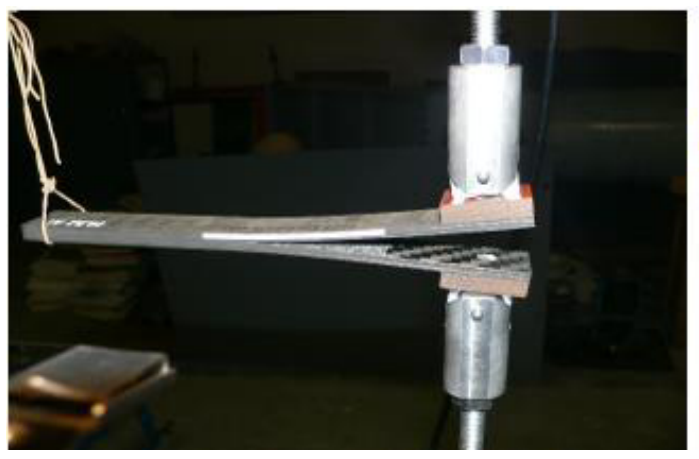

a)

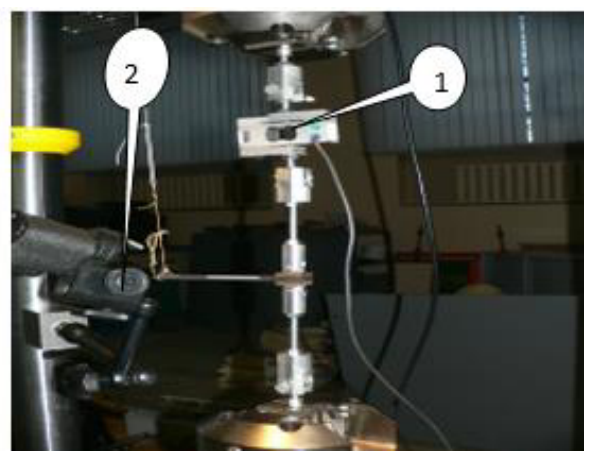

b)

Fig. 1. Basic fragments of test setup: DCB sample (a), items of loading and measurement(b): HBM S2M meter (1), microscope(x10) (2)

But for the actual material the DCB sample behavoir in this test is more complicated. The typical original record of a function 'extension/force' is presented in the Fig.2. It can be seen: 
1. In general, the record of process of a DCB sample loading can be sheared to ascending and descending branches by the point of maximal force. But the loading function for each branch is not monotonic.

2. At the first step of ascending branch the monotonic increasing of external force is observed with approximately linear function 'extension/force'. First interruption of monotonicity of the loading function occurs at the value of force about $70 \%$ of maximal force: small down jump of force at practically constant extension. At the further increasing of extension, the number of down-jumps increases, and the nonlinearity of the function 'extension / force' becomes more and more noticeable.

3. For all points of jump-type interruption of crack growth in the ascending branch the strain energy realese rate can increase only at increasing of load. It means that the crack résistance function also increases.

4. After reaching of maximal force there is beginning of a descending branch of loading. Here the similar features of a crack growth are observed. Only the crack length jump-type increments are significantly larger.

5.There is remaining extension after full unloading of a sample

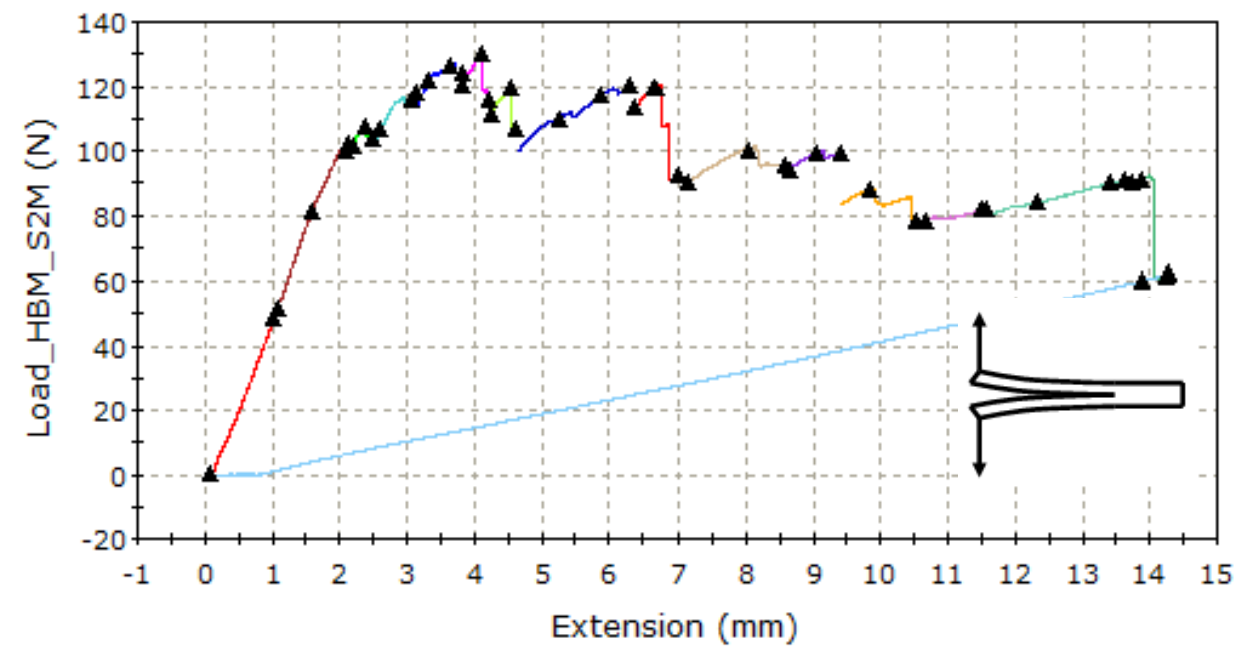

Figure 2. Original record of Instron measurement system at the test of DCB sample of layered composite

The mentioned above features of process of DCB sample destruction can be considered more details using the crack length/force and the functions 'crack length/extension' (Fig.3 and Fig.4). Note that crack length was basically defined by compliance method with correction using results of direct measurement and correction item of Standard [16] for accounting of effect of rotation of cross-sections of a sample at the front of delamination.

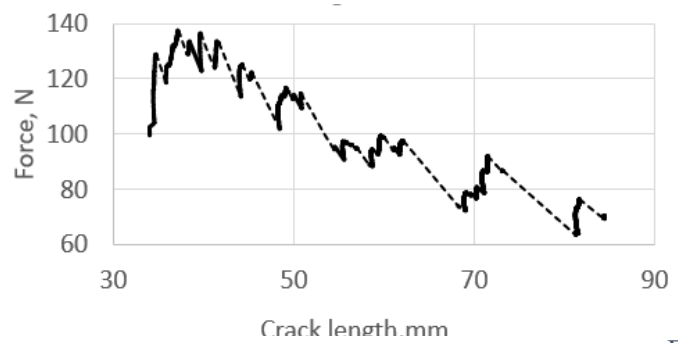

Figure 3. The force via crack length

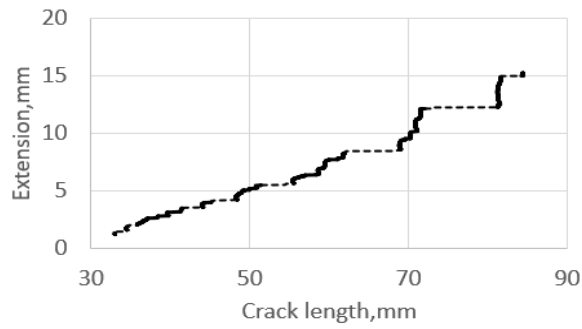

Figure 4. The extension via crack length 
The general conclusion here is: the crack resistance can not be some constant of material, but is some function of crack increment. At least, the initial portion must be, in average, the ascending function. Cross-ply cracking, local delamination, inhomogeneity of the mechanical characteristics of the composite components, structural defects cause random fluctuations of a crack

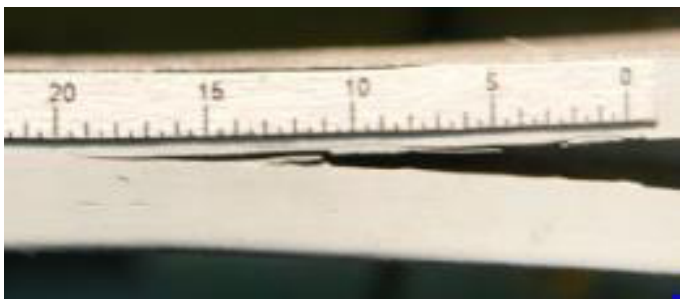

Figure 5. View of real structure of a crack surface resistance (for instance, see Fig.5). In the picture of a lateral side of the DCB sample of considered layred composie can see that crack surfaces relief is very complicated.

\section{Briefly on the model of a crack quasi-static growth}

\subsection{Basic assumptions}

Small-scale cohesive zone [3] is basic restriction of proposed model in a frame of which two key assumptions are accepted:

1. The cohesive zone is represented by the Dugdale's model[21] in the option of small-scale dimensions. It means the size $r$ of cohesive zone is much less than the length of the crack ( $r \ll l)$ and

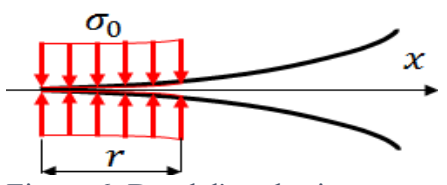

Figure 6. Dugdal's cohesive zone

$$
r=\frac{\pi}{8} \frac{K^{2}}{\sigma_{0}^{2}}
$$

where $K$ is stress intensity factor (SIF) that defines the stress-strain state close a crack tip, and $\sigma_{0}$ defines the intensity of cohesive forces (for elastic-perfectly plastic material is equal to material yield stress). Here a stress intensity factor $K$ corresponds to actual crack length $l$. In term of the strain energy release rate $G$ the equation (1) can be expressed as follow:

$$
r=\frac{\pi}{8} \frac{G E}{\sigma_{0}^{2}}
$$

where $E$ is the generalized modulus of elasticity (defined by the type of the stress state).

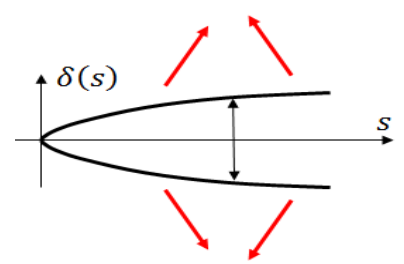

Figure 7. Crack opening model

Thus, the cohesive zone is presented as a crack extension, the sides of which are loaded by uniformly distributed direct stresses $\sigma_{0}$.

2. The crack opening function $\delta(s)$ within the cohesive zone under the action of an external load only is described by the first (dominant) term of decomposition of displacement at crack tip into a power series

$$
\delta(s) \approx \frac{8 K\left(l_{e f}\right)}{E} \sqrt{\frac{s}{2 \pi}}
$$

where $s$ is the distance of the cohesive zone boundary point from the crack tip, and $t$ is the constant thickness a plate (further this type of a body is considered). Note that here a stress 
intensity factor $K\left(l_{e f}\right)$ corresponds to effective crack length $l_{e f}=l+r$ (including size of a cohesive zone). At the assumption of small-scale size of cohesive zone, the linearized relation between effective $K\left(l_{e f}\right)$ and the stress intensity factor $K$ for a crack of actual length $l$ is represented by the follow equation

$$
K\left(l_{e f}\right) \approx K+\frac{\partial K}{\partial l} r=K\left(1+\frac{\pi}{16 \sigma_{0}^{2}} \frac{\partial K^{2}}{\partial l}\right)
$$

The corresponding relation for the strain energy release rate is

$$
G\left(l_{e f}\right) \approx G+\frac{\partial G}{\partial l} r=G\left(1+\frac{\pi}{8} \frac{E}{\sigma_{0}^{2}} \frac{\partial G}{\partial l}\right)
$$

\subsection{Energy balance and the general equation of a crack quasi-static growth}

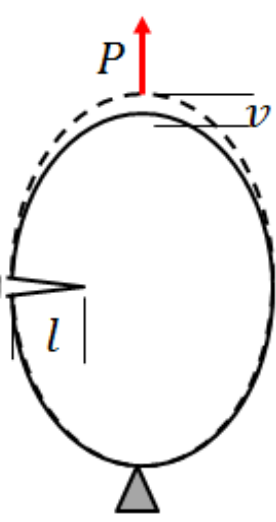

Figure 7.The scheme of generalized loading

The model of quasi-static process of crack propagation assumes that this process develops slowly and continuously, and in each position the system internal and external parameters satisfy to equation of energy balance. In terms of the generalized force $P$ and corresponding the generalized displacement $v$ (Fig.7) this equation in differential form can be presented as follow:

$$
\frac{1}{2 t} P \frac{d v}{d l}-\frac{1}{2 t} v \frac{d P}{d l}=G_{c}^{*}(\Delta l)
$$

In general case of quasi-static crack growth, the $G_{c}^{*}(\Delta l)$ is equal to the critical dissipation energy rate and further is named as the total crack resistance. Total energy of dissipation includes: 1) energy of cohesive zone development and 2) surface energy of new surface of a crack. The dissipation energy rate coincidences with the fracture toughness only, if $d P / d l=0$. The fracture toughness is a mechanical constant of material and here is assigned by $G_{c}$. In all other possible states of equilibrium, the dissipation energy rate depends on parameters of this state, particularly, a crack size increment $\Delta l$ in respect to size of a crack at initial unloaded state.

Eq. 6 can be simply transformed to follow:

and more detailed in Eq. 8

$$
\frac{1}{2 t} P \frac{\partial v}{\partial l}+\frac{1}{2 t}\left(P \frac{\partial v}{\partial P}-v\right) \frac{d P}{d l}=G_{c}^{*}(\Delta l) .
$$

$$
\frac{1}{2 t} P \frac{\partial v_{e}}{\partial l}+\frac{1}{2 t} P \frac{\partial v_{r}}{\partial l}=G_{c}^{*}(\Delta l)-\frac{1}{2 t}\left(P \frac{\partial v_{r}}{\partial P}-v_{r}\right) \frac{d P}{d l}
$$

Eq. 8 can be named as the general equation of a crack quasi-static growth.

Here the generalized displacement $v$ is equal to sum of two components:

$$
v=v_{e}+v_{r}
$$

The elastic component $v_{e}$ is linearly related with the generalized force and completely defined by elastic compliance of a body with the actual crack length $l$. This component disappears after full elastic unloading. The cohesive zone appearance at the crack tip causes additional displacement $v_{r}$, which remains after full elastic unloading and is associated with the effect of material physical nonlinearity. This can be called as the residual component of generalized displacement.

The sum of both items in left side of Eq. 8 is equal to total strain energy realize rate $G=$ $G_{e}+G_{r}$. Easy see that right side of Eq.8 is equal to the crack-resistance function, so called, the $R$-curve. It is equal to a difference between total dissipation energy rate and an item which can be associated with energy rate of cohesive zone development. So, the $R$-curve general equation is: 


$$
R=G_{c}^{*}-\frac{1}{2 t}\left(P \frac{\partial v_{r}}{\partial P}-v_{r}\right) \frac{d P}{d l}
$$

The general analysis of $R$-curve allows to obtain some important conclusions. Only in two specific cases $\left.R=G_{c}^{*}: 1\right)$ perfectly brittle material, or 2) critical energy balance that corresponds to condition $d P / d l=0$. In all other cases $R \neq G_{c}^{*}$. The Eq.8 shows, if $d P / d l>$ 0 (ascending branch of $R$-curve), then the crack-resistance $R<G_{c}^{*}$. But if $d P / d l<0$ (descending branch of $R$-curve), then the crack-resistance $R>G_{c}^{*}$.

\subsection{The invariant equation of a crack quasi-static growth}

Using basic assumptions of the developed model, the general equation of a crack quasi-static growth (Eq.8) can be represented in invariant form in respect to the geometrical configuration of a body with crack and its loading mode. This equation can be simplified by eliminating second-order terms of smallness in respect of the critical size of the plastic zone. As a result, the normalized invariant form of equation of a crack quasi-static growth is as follow:

$$
\frac{d \bar{P}}{d \bar{l}}=\frac{1-\bar{G}\left(1+\frac{4}{3} \bar{r}_{c} \frac{\partial \bar{G}}{\partial \bar{l}}\right)}{\frac{4}{3} \bar{r}_{c} \frac{\bar{G}^{2}}{\bar{P}}} .
$$

where $\bar{P}=P / P_{c}, \bar{l}=l / l_{0}, \bar{r}_{c}=r_{c} / l_{0}$ and $\bar{G}=G / G_{c}$.

More detailed description of Eq.11, resolving method, properties and examples of solution for some configurations of a cracked body can find in $[18,19]$.

\section{The model of a crack quasi-static growth for variable crack resistance}

\subsection{Modified invariant equation}

Eq.11 corresponds to assumption that the total crack resistance is constant which equal to the interlaminar fracture toughness $G_{c}^{*}=G_{c}$. But in general case of the total crack resistance is variable

$$
G_{c}^{*}=\delta G_{c}
$$

where $\delta$ is a dimensionless function of crack increment $\Delta l$. In general, it is random function, at least, with two scalar paranerers (position and scale).

In this case Eq. 11 transforms to follow:

$$
\frac{d \bar{P}}{d \bar{l}}=\frac{\delta-\bar{G}\left(1+\frac{4}{3} \delta \bar{r}_{c} \frac{\partial \bar{G}}{\partial \bar{l}}\right)}{\frac{4}{3} \delta \bar{r}_{c} \frac{\bar{G}^{2}}{\bar{P}}} .
$$

\subsection{Solution for DCB sample with the variable crack resistance}

For DCB sample

$$
\bar{G}=\bar{P}^{2} \bar{l}^{2}
$$

In this case Eq.13 can be converted to follow:

$$
\frac{d \bar{P}}{d \bar{l}}=\frac{\delta-\bar{P}^{2} \bar{l}^{2}\left(1+\frac{8}{3} \delta \bar{r}_{c} \bar{P}^{2} \bar{l}\right)}{\frac{4}{3} \delta \bar{r}_{c} \bar{P}^{3} \bar{l}^{4}}
$$

For numerical example the simple analytical expression of the coefficient $\delta$ of total crack resistance is selected as follow: 


$$
\delta=\left\{\begin{array}{c}
0, \quad \text { at the } l<l_{0} \\
1+\alpha \sin \left(2 \pi \bar{x} \bar{l}_{0}+\varphi_{0}\right), \quad \text { at the } l>l_{0}
\end{array}\right.
$$

Three independent parameters define the shape of this function: a dimensionless amplitude $\alpha$ (as a part of the mean value of the fracture toughness $G_{c}$ ), a spatial period $L$ and a phase angle $\varphi_{0}$. In the equation (14) also $\bar{l}_{0}=l_{0} / L, \bar{x}=\left(l-l_{0}\right) / l_{0}, x=l-l_{0}=\Delta l$.

Calculation results is shown below. The loading diagram is presented in the Fig.8. The

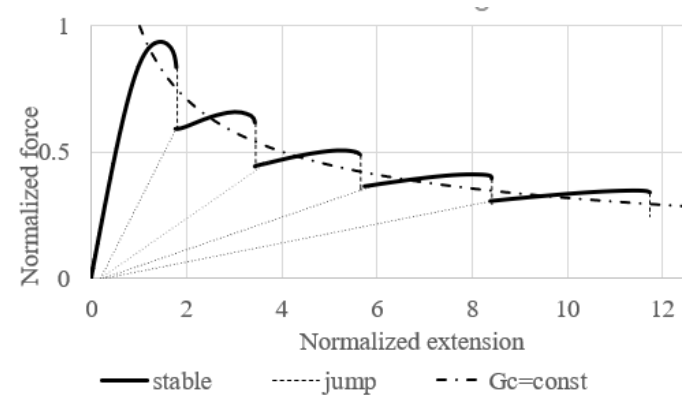

Figure 8. Predicted loading diagramm steps of loading those are predicted by model of quasy-static crack growth are shown by solid fragments of loading diagram. The jump-type parts of loading process are shown by vertical dash lines and correspond to dynamic crack propagation. Bottom point of each of those lines is theoretical position of a crack arrest: here all parameters of system satisfy to the equation of energy balance. The dash-dot line corresponds quasy-static crack growth at constant crack resistance of material.

In respect of prediction of a crack behavoir the loading control law should be considered together with the process of quasy-static crack growth. The quasy-static crack growth in terms of 'crack length/force' and 'crack length/extension' are shown in Fig.9 and Fig.10 respectively.

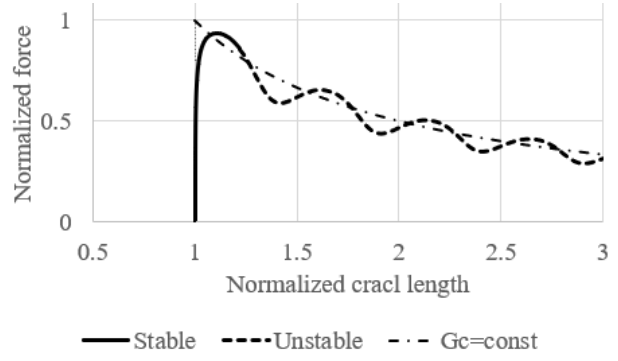

Figure 9. Crack length/force diagramm

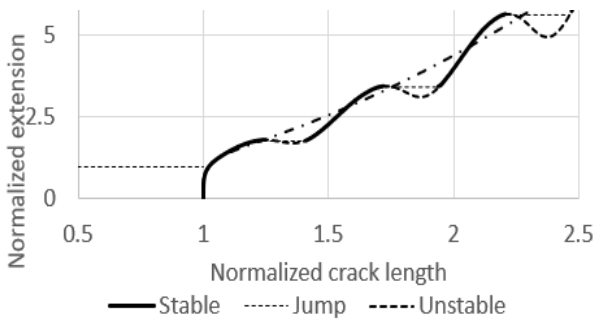

Figure 10. Crack length/extension diagramm

At the force control process of a crack growing will be for maximal force only(Fig.9). At the extension control the portions of stable crack growth (bold line) alternate with a crack jump-type increment of crack length (horizontal dash lines in Fig.10).

\section{Final outcome of experimental research}

Using Eq.7 and test results represented by Instron record (Fig.2) and its derivatives (Fig.3 and Fig.4) some final results were obtained. In Fig. 11 is present the total crack resistance of given layered composite. It is seen that solid lines of diagramm 'crack length/total crack resistance' correspond to stable development of a crack. The dashed lines correspond to unstable crack growing. Loading mode with control of displacement allows to estimate the mean rate of crack propagation as follow:

$$
\dot{l}=\frac{d l}{d t}=\dot{v} \frac{d v}{d l}
$$




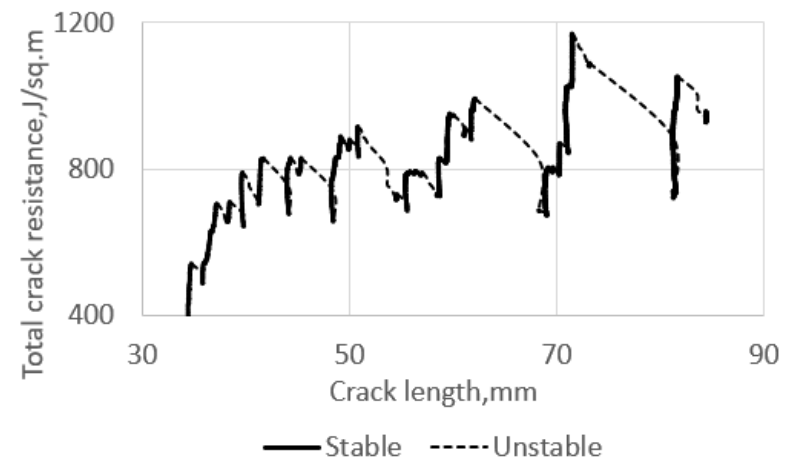

Figure 11. Total interlaminar crack resistance

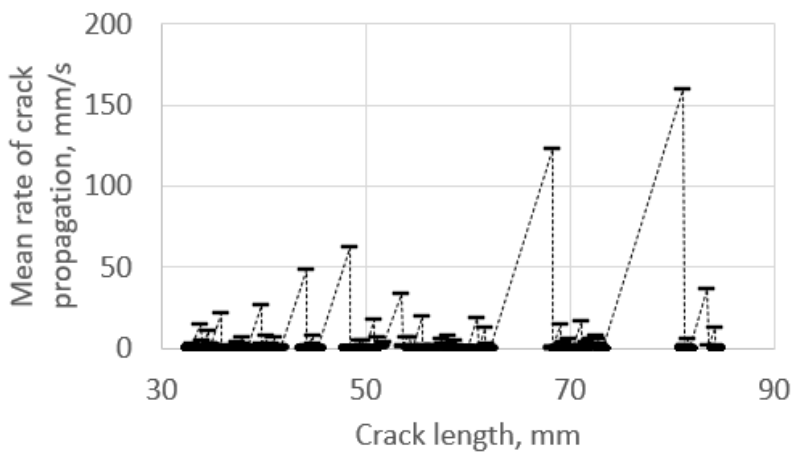

where $\dot{v}=5 \mathrm{~mm} / \mathrm{min}$ is the extension constant rate, but a derivative $d v / d l$ was obtained by numerical differentiating of corresponding function (Fig.4).

In the Fig.12 the diagram of a crack growth rate is presented. By the marker (horizontal bar) is shown mean value of a crack growth rate and the two dash line connected with marker indicate ends of corresponding increment of a crack length. It is seen that for the jump-type crack increments the mean rate of crack growth is large in respect of description crack propagation as a quasi-static process.

Using the total crack resistance function (Fig.11) and Eq.10, the crack resistance $R$-curve can be obtained. For this porpose the member that is associated with the part dissipation energy rate for a cohesive zone development $G_{r}$ for the DCB sample is as follow:

Figure12. Diagramm of a crack rate

$$
G_{r}=\frac{1}{2 t}\left(P \frac{\partial v_{r}}{\partial P}-v_{r}\right) \frac{d P}{d l}=\frac{\pi}{6} \frac{E}{\sigma_{0}^{2}} \frac{P^{3} l^{4}}{D^{2} t^{2}} \frac{d P}{d l}
$$

where $D=E t h^{3} / 12$ is the cylindrical stiffenes of of rectangular cross-section of an arm of

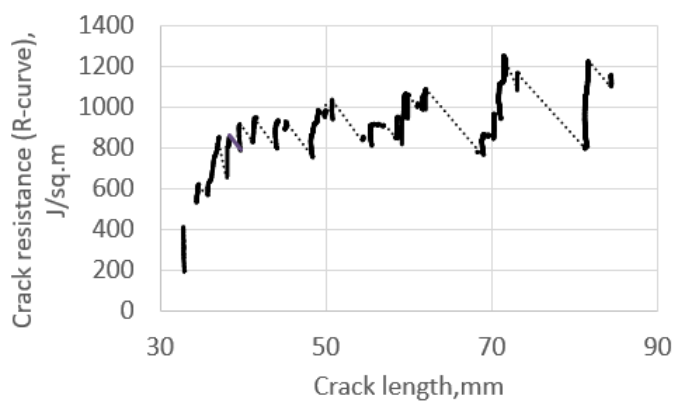

Figure 13. Crack resisrance ( $R$-curve) the DCB sample of a width $t$ and a height $h$.

The cohesive zone development energy rate was calculated using (18) and substructed from the total dissipation energy rate. As a result, the crack resistance $R$-curve was presented by final diagramm (Fig.13) by portions corresponded to stable steps of crack growth.

$R=G_{c}^{*}-G_{r}$

\section{Brief discussion and conclusions}

Using the model of quasi-static crack growth, the general regularities of the interlaminar crack resistance of a layered composite in mode 1 were studied. The main attention was 
focused on the variability of crack resistance associated, on the one hand, with the continuity of the fracture process, and, on the other hand, with the inhomogeneity of the material structure, which causes local random deviations from the average characteristics of the material. Two main characteristics were used: the rate of energy dissipation and the critical strain energy realise rate or briefly the function of crack resistance (R-curve). The rate of energy dissipation is a characteristic of total crack resistance since it combines the loss of total potentional energy to create an unit of new surface associated with two main causes: 1) energy for development of the cohesive zone and 2) energy of the new surface of a crack. The experiment was carried out on standard DCB samples of carbon / epoxy layred composite. Analysis of the experimental results and their comparison with the simulation results allowed to obtain a number of significant conclusions, which are discussed below.

1) The developed model of quasi-static crack growth, which is based on the concept of a small-scale cohesive zone acepted in fracture mechanics and on the assumption of the continuity of crack growth at increasing load, makes it possible to adequately describe the features of the behavior of a body with a crack at the restrictions of a model..

2) A typical situation is when, immediately before the crack jump, the derivative of the force per the crack length is not zero $d P / d l \neq 0$. This is clearly seen from Fig. 4. Mostly $d P / d l>$ 0 , i.e. the crack jump occures during movement at the ascending branch of stable crack growth. First of all, this indicates that there is a discontinuity of the function of crack resistance or its derivative per crack length. On the other hand, $d P / d l>0$ means that energy dissipation is associated with the action of both mechanisms: expansion of the cohesion zone and growth of the crack surface.

3) The diagrams of the crack resistance characteristics of the composite obtained from the experiment (total crack resistance, determined by the rate of energy dissipation, and the the crack resistance function or $R$-curve) are shown in Fig. 11 and Fig. 13 as portions of stable crack growth. The lower point of each portion determines the value of the corresponding crack resistance parameter at the moment of crack arrest after the previous jump. The upper point of each portion shows the maximum value of the crack resistance parameter at the corresponding position.

4) The transition from one portion of stable growth to the next occurs abruptly with an average crack growth rate shown in Fig. 12. It is seen, this rate is significant and can have a important impact on the determination of crack resistance. One can only assume that it in the interval between the maximum of crack resistance and its value at the crack arrest.

5) From the graph of $R$-curve Fig. 12 can see that at crack length more than 35 the crack resistance of crack arrest is practically constant and approximately equal to $800 \mathrm{~J} / \mathrm{sq} . \mathrm{m}$ and can be called as the cack arrest interlaminar fracture toughness.

\section{References}

1. Griffith A.A. Phil. Trans. Roy. Soc., A 221, 163-198 (1921).

2. Irwin, G.R. Plastic zone near a crack and fracture toughness. Proc. 7th Sagamore Conf., IV-63 (1960)

3. G. I. Barenblatt. Adv. Appl. Mech. 7, 55-129 (1962).

4. D.Broek. Elementary engineering fracture mechanics(1982) https://doi.org/10.1007/978-94-011-9055-8

5. D.Broek. in: Aerospace Proceedings, 1966. Royal Aeronautical Society Centenary Congress in Conjunction with Fifth Congress of the International Council of the Aeronautical Sciences. 811-835. Macmillan, London, (1967) 
6. G.P. Cherepanov1968.. PMM . 32, 6 (1968)

7. K.N Raju. Int. J. Fract. Mech. (1969)

8. Rice, James R. in: Liebowitz, H. (Ed.), Fracture: An Advanced Treatise (Vol. 2, Mathematical Fundamentals). Academic Press, N.Y., pp. 191-311, (1968).

9. M.P.Wnuk. Int. J. Fract. Mech. 7 (1971)

10. Rice, J.R. in: Mechanics and Mechanisms of Crack Growth, Cambridge, England, 14 - 6 April 1973. pp. 1-34 (1975).

11. Rice, J.R. in: American Society of Mechanical Engineers, Applied Mechanics Division, AMD. (1976) https://doi.org/10.1201/b19173-12

12. Rice, J.R. J. Mech. Phys. Solids 26, 61-78 (1978). https://doi.org/10.1016/0022$\underline{\text { 5096(78)90014-5 }}$

13. Rice, J.R., Sorensen, E.P. J. Mech. Phys. Solids 26, 163-186 (1978). https://doi.org/10.1016/0022-5096(78)90007-8

14. J. Hutchinson and P. Paris. in: Elastic-Plastic Fracture, ed. J. Landes, J. Begley, and G. Clarke (West Conshohocken, PA: ASTM International), 37-64 (1979).

15. M.P.Wnuk, T.Mura. Int. J. Eng. Sci. 19, 1517-1527 (1981)

16. Nairn, J.A. Int. J. Fract. 76, 155-167 (2009). https://doi.org/10.1007/s10704-0099338-3

17. Nairn, J.A. Eng. Fract. Mech. 72, 961-979 (2005) https://doi.org/10.1016/j.engfracmech.2004.08.006

18. V.Pavelko. Key Engineering Materials, 827, 312-17 (2020) https://doi.org/10.4028/www.scientific.net/KEM.827.312

19. V.Pavelko. AIP Conference Proceedings 2309, (2020) DOI:10.1063/5.0034981

20. ASTM D5528 Standard Test Method for Mode I Interlaminar Fracture Toughness of Unidirectional Fiber-Reinforced Polymer Matrix Composites

21. Dugdale, D.S. J.of the Mechanics and Physics of Solids. 8, 100-108 (1960). https://doi.org/10.1016/0022-5096(60)90013-2 